\title{
Detection scheme for white-light interferometric sensors
}

\author{
H. R. Giovannini, D. Yeddou, and S. J. Huard \\ Laboratoire d'Optique des Surfaces et des Couches Minces, U.R.A. 1120 Centre National de la Recherche Scientifique, \\ Ecole Nationale Supérieure de Physique de Marseille, 13397 Marseille Cedex 20, France
}

M. R. Lequime

Optics and Optoelectronics Division, Bertin \& Cie, 230 rue Frédéric Joliot, Z.I. Les Milles, B.P. 22000, 13791 Aix-en-Provence Cedex 3, France

\section{Froehly}

IRCOM, 123 Avenue Albert Thomas, 87060 Limoges Cedex, France

Received June 22, 1993

\begin{abstract}
Static interferometers whose optical path difference is not constant over the field of interferences are well adapted to demodulate sensing interferometers in the coherence multiplexing method. In this configuration the correlation peak can be spatially recorded on a linear detector array. However, the determination of the position of the peak maximum amplitude is often difficult because of the spatial sine amplitude modulation. This drawback can be eliminated by using a grating interferometer as a demodulator. In this case it is possible to record, on the detector, only the envelope of the correlation peak.
\end{abstract}

Fiber-optic sensors are useful for industrial applications in perturbed environments. One of the main advantages of these devices, as a result of the use of fiber optics, is that the information (modulation of the optic signal) about the measurand can be transmitted over long distances. However, the method used to encode the parameter to be measured and to decode it must be carefully chosen to preserve this advantage. Thus it is necessary that the fiber transmission of the optical signal be made independently of the losses, along the fiber link. Coherence multiplexing ${ }^{1,2}$ appears to be a well-adapted method to meet this requirement., ${ }^{3,4}$ We report, together with a theoretical analysis of the device, the experimental results obtained when a grating interferometer ${ }^{5}$ is used to demodulate an interferometric sensor (simulated by a Michelson interferometer).

In the coherence multiplexing method, a broadband light source illuminates an interferometric sensor whose optical path difference (OPD), denoted $\Delta_{s}$, is much greater than the source coherence length, denoted $l_{c}$. The spectral distribution $F_{1}$ of the transmitted light can be written as

$$
F_{1}(\sigma)=\frac{1}{k} F_{0}(\sigma)\left[1+\cos \left(2 \pi \sigma \Delta_{s}\right)\right],
$$

where $F_{0}(\sigma)$ is the spectral distribution of the incident light and $k$ is an integer, equal to 2 for the Michelson interferometer and to 4 for a birefringent interferometer (unpolarized incident light). The spectral distribution of the transmitted light exhibits a comb spectrum, and the frequency of the combs is related to $\Delta_{s}{ }^{6,7}$ Variations of the line transmissions do not influence the frequency of the combs. Therefore this encoding method permits the information on $\Delta_{s}$ to be transmitted with less dependence on the line losses than in the case with an intensity encoding scheme. To recover $\Delta_{s}$, we launch the light through a demodulation interferometer ${ }^{8}$ whose OPD is denoted $\Delta_{d}$ and sent to a photodetector, which gives a signal proportional to $R$ with

$$
\begin{aligned}
R= & \frac{1}{k k^{\prime}} \int_{\sigma} F_{0}(\sigma)\left[1+\cos \left(2 \pi \sigma \Delta_{s}\right)\right] \\
& \times\left[1+\cos \left(2 \pi \sigma \Delta_{d}\right)\right] \mathrm{d} \sigma,
\end{aligned}
$$

where $k^{\prime}$ is the same kind of parameter as $k$ in Eq. (1).

With the assumption that the source spectrum is symmetric around the central wave number $\sigma_{1}$, $R$ appears to be composed of four terms: a continuous background, a central peak, obtained for $\Delta_{d}<l_{c}$, and two correlation peaks, whose envelopes are maximum for $\Delta_{d}= \pm \Delta_{s}$. The peaks are modulated by a sine function whose phase $\phi$ is equal to $2 \pi \sigma_{0}\left(\Delta_{s}-\Delta_{d}\right)$. The measurement of $\phi$ permits $\Delta_{s}$ to be recovered. Although this method leads to the most accurate measurement, it requires rather complex systems. ${ }^{9}$ Another way to recover $\Delta_{s}$ is to determine the value of $\Delta_{d}$ corresponding to the maximum correlation peak amplitude, $\Delta_{d M}$; thus we have $\Delta_{d M}=\Delta_{s}$. This can easily be achieved by choice of a static demodulator whose phase is not constant over the interference field (a Michelson interferometer with one tilted mirror ${ }^{10}$ or a Babinet compensator in polarized light $\left.{ }^{11}\right)$. Then the light is analyzed by a linear detector array. These devices are static and their setup is easy, but, because of the chromatic dispersion of $\Delta_{s}$ and $\Delta_{d}$, the maximum amplitude of the correlation peak may not correspond to a maximum of the recorded signal. ${ }^{12}$ This means that it is necessary to rebuild the peak envelope numerically to recover $\Delta_{s}$. We can avoid this problem by using, as a demodulator, a grating interferometer whose scheme 


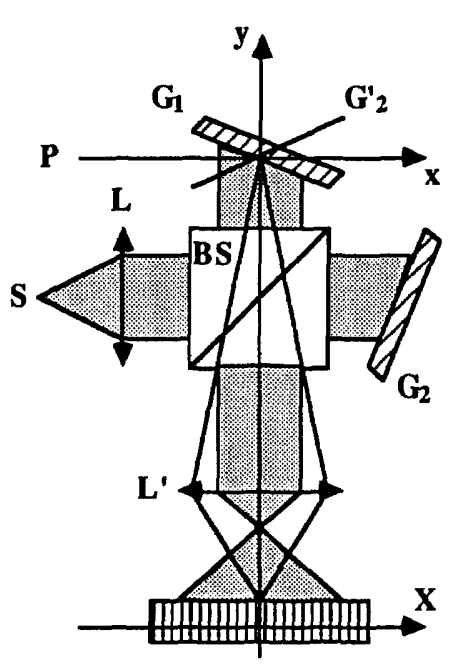

Fig. 1. Configuration of the grating interferometer. $\mathrm{G}_{2}{ }^{\prime}$, the image of $\mathrm{G}_{2}$ through BS.

is shown in Fig. 1. The light from the point source is collimated by lens $L$; the two gratings, $G_{1}$ and $G_{2}$, are in a Littrow mount in the first order for the wave number $\sigma_{0}$ and are symmetric with respect to the beam splitter, BS. After diffraction by the gratings, the direction of the wave surface depends on the wave number $\sigma$. Then the OPD in the bisecting $\mathrm{P}$ plane can be written as $^{5}$

$$
\Delta_{d}(\sigma, x)=2 \alpha(\sigma) x,
$$

where $\alpha$ is the angle between the direction of the diffracted beam and the $O y$ axis. With an angular dispersion $D=\sigma(\mathrm{d} \alpha / \mathrm{d} \sigma)$, we have $\alpha(\sigma)=D(\sigma-$ $\left.\sigma_{0}\right) / \sigma$. Denoting $p$ the grating spacing and $i_{0}$ the angle of the $O y$ axis and the normal to the grating, we have

$$
\Delta_{d}(x, \sigma)=\frac{2 x}{p \sigma \cos i_{0}}-4 x \tan i_{0} .
$$

To simplify the analytical developments, we assume that the spectral distribution of the source, $F_{0}(\sigma)$, is a gate function, centered on $\sigma_{1}$, whose width is denoted $\Delta \sigma$. The image of the $\mathrm{P}$ plane is formed by objective $L^{\prime}$ on a linear detector array, with a magnification of $G$. Obviously, $X=G x$. In this case, the recorded signal is proportional to $R^{\prime}(X)$, with

$$
R^{\prime}(X)=1+A+\frac{1}{2} B_{1}+\frac{1}{2} B_{2},
$$

where

$$
A=\frac{\sin U}{U} \cos \left[4 \pi \frac{X}{G}\left(\frac{1}{p \cos i_{0}}-2 \sigma_{1} \tan i_{0}\right)\right],
$$

$$
\begin{aligned}
& B_{1}=\frac{\sin \left(U-\pi \Delta_{s} \Delta \sigma\right)}{U-\pi \Delta_{s} \Delta \sigma} \\
& \quad \times \cos \left[4 \pi \frac{X}{G}\left(\frac{1}{p \cos i_{0}}-2 \sigma_{1} \tan i_{0}\right)+2 \pi \sigma_{1} \Delta_{s}\right]
\end{aligned}
$$

$$
\begin{gathered}
B_{2}=\frac{\sin \left(U+\pi \Delta_{s} \Delta \sigma\right)}{U+\pi \Delta_{s} \Delta \sigma} \\
\times \cos \left[4 \pi \frac{X}{G}\left(\frac{1}{p \cos i_{0}}-2 \sigma_{1} \tan i_{0}\right)-2 \pi \sigma_{1} \Delta_{s}\right], \\
U=4 \pi \frac{X}{G} \Delta \sigma \tan i_{0} .
\end{gathered}
$$

Thus $R^{\prime}(X)$ is composed of (1) a continuous background, (2) a central peak (described by $A$ ), centered on $X=0$, and (3) two secondary peaks (described by $B_{1}$ and $\left.B_{2}\right)$, centered on $X= \pm \Delta_{s} G /\left(4\right.$ tan $\left.i_{0}\right)$. The amplitudes of the three peaks are spatially modulated by a sine function. When $\sigma_{0}=\sigma_{1}$ (the gratings are in a Littrow mount for the central wave number of the source), we have

$$
\frac{1}{p \cos i_{0}}-2 \sigma_{0} \tan i_{0}=0,
$$

the spatial modulation disappears, and the amplitude is proportional to $\cos \left(2 \pi \sigma_{0} \Delta_{s}\right)$ for the two secondary peaks. The value of $X$ corresponds to the maximum amplitude of one of the two secondary peaks, $X_{M}$, gives the value of $\Delta_{s}$.

In our experiment, a Michelson interferometer was used as a sensor and coupled to the grating interferometer. A schematic diagram of the experimental setup is shown in Fig. 2. The source used in our experiment is a light-emitting diode (LED) whose emission spectrum is centered on $\lambda_{1}=850 \mathrm{~nm}$, with a coherence length $l_{c} \approx 15 \mu \mathrm{m}$. All the optical fibers used are standard 100/140 multimode fibers. The gratings are carved with a spacing $p=3.3 \mu \mathrm{m}$, blazed for $\lambda=600 \mathrm{~nm}$. The linear array is an array of 1024 photodiodes. The image of the $\mathrm{P}$ plane is formed with a magnification $G \approx 12$. The translation of one mirror of the Michelson interferometer can be done with an accuracy of $1 \mathrm{~nm}$ thanks to a piezoelectric transducer. In all the following figures showing the experimental results, $R(X)$ is represented as a function of time, because of the use of

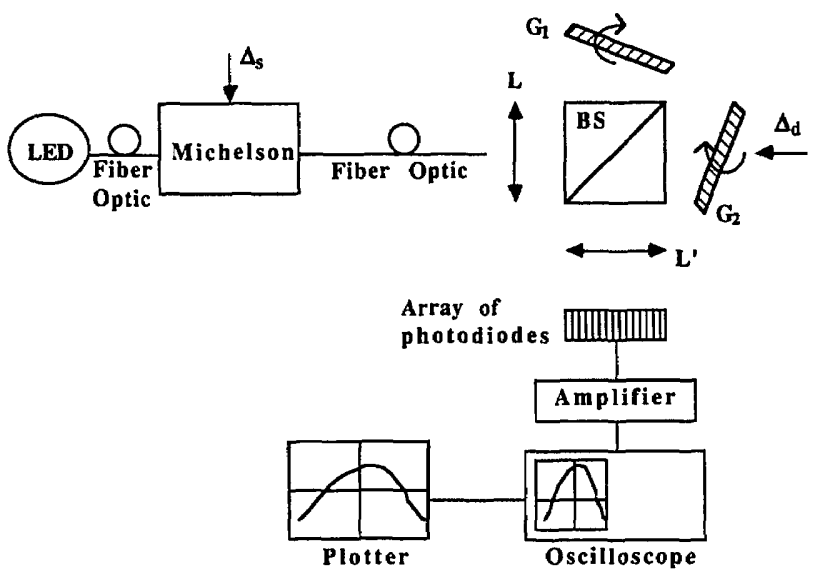

Fig. 2. Experimental setup. Gratings $G_{1}$ and $G_{2}$ can be rotated by the same angle to change the wave number of the Littrow mount. 
(a) $\mathrm{CH} \quad 0.1 \mathrm{~V} \quad 2 \mathrm{mS}$

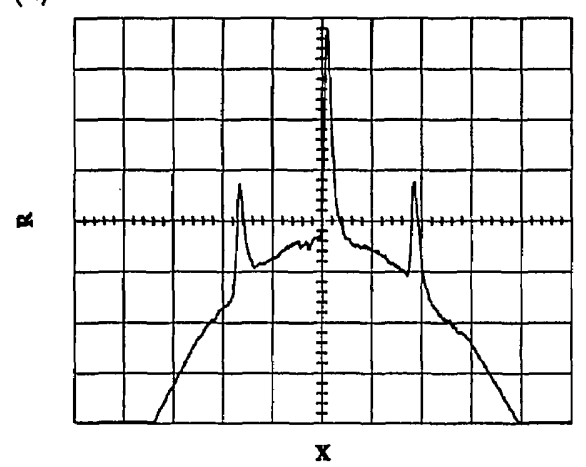

(b) $\quad$ CH1 $\quad 0.1 \mathrm{~V} \quad 2 \mathrm{~ms}$

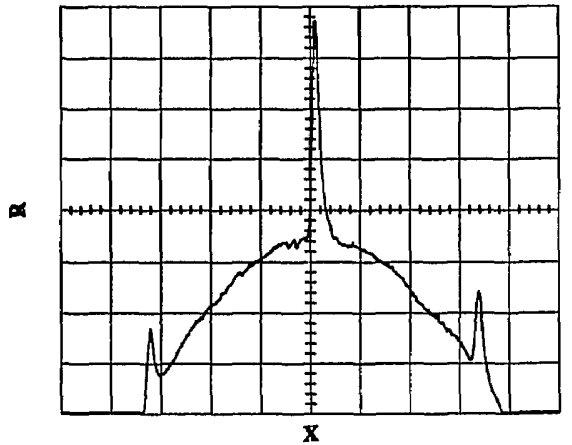

Fig. 3. Recorded signals $R(X)$ : (a) $\Delta_{s}=374 \mu \mathrm{m}$, (b) $\Delta_{s}=690 \mu \mathrm{m}$. The gratings are in a Littrow mount for $\lambda_{0}=850 \mathrm{~nm}$.

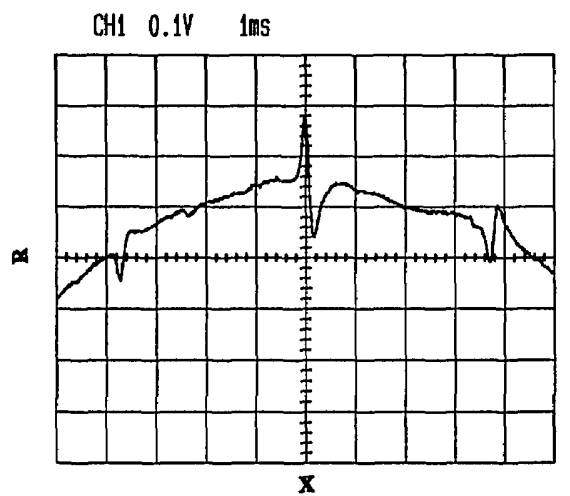

Fig. 4. Recorded signal $R(X)$ for $\Delta_{s}=690 \mu \mathrm{m}$. The gratings are in a Littrow mount for $\lambda_{0} \approx 820 \mathrm{~nm}$.

an oscilloscope to record the signal at the output of the amplifier.

Figure 3 shows the recorded signals $R(X)$ for two values of $\Delta_{s}$ when the gratings of the grating interferometer are in a Littrow mount for the same wave number as the central wave number of the source. As expected, the two secondary peaks are symmetric around $X=0$, where the central peak amplitude is maximum and the sine spatial modulation amplitude is eliminated. One can see the displacement of the secondary peaks corresponding to a variation of $\Delta_{s}$.
When $\sigma_{0} \neq \sigma_{1}$, the amplitudes of the three recorded peaks are spatially modulated, as shown in Fig. 4, in which the gratings of the demodulator are in a Littrow mount for $\lambda_{0} \approx 820 \mathrm{~nm}$. One can see that there is perfect agreement between the theory and the experimental results.

In conclusion, we have demonstrated that a grating interferometer can be used to demodulate white-light interferometric sensors. With simple data processing, the resolution performance of our experimental device for the measurement of the OPD in the sensor is better than $2 \mathrm{~nm}$ over a $2-\mathrm{mm}$ range. It is possible to increase these performances by changing the magnification $G$ and by choosing an array with more detectors. Moreover this device is well suited to demodulation of several sensors. In this case, the OPD variation of each sensor is related to the displacement of the corresponding secondary peaks, and the bandpass of the measurement is limited only by that of the photodiode array. However, because of the term $\cos \left(2 \pi \sigma_{0} \Delta_{s}\right)$ in Eqs. (6b) and (6c), the amplitude of the two secondary peaks may be null, thus making the measurement impossible. To solve this problem, one can add in either arm of the demodulator a retarder designed to introduce a retardation equal to $\pi / 2$. Thus two other peaks, whose amplitudes are proportional to $\sin \left(2 \pi \sigma_{0} \Delta_{s}\right)$, can be recorded, making the measurement still possible.

The authors are pleased to acknowledge technical help and valuable suggestions from $\mathrm{H}$. Akhouayri.

\section{References}

1. J. P. Goedgebuer, H. Porte, and A. Hamel, IEEE J. Quantum Electron. QE-23, 1135 (1987).

2. F. Farahi, T. P. Newson, J. D. C. Jones, and D. A. Jackson, Opt. Commun. 65, 319 (1988).

3. Ph. Dabkiewicz and R. Ulrich, in Proceedings of the 3rd European Fiber Optic Communications and Local Area Networks Exposition (Information Gatekeepers, Boston, Mass., 1985), pp. 212-217.

4. C. Delisle and P. Cielo, Can. J. Phys. 54, 2322 (1976).

5. P. Connes, Rev. Opt. 38, 157, 416 (1959); 39, 402 (1960).

6. B. Colombeau, M. Vampouille, and C. Froehly, in Progress in Optics XX, E. Wolf, ed. (Elsevier, Amsterdam, 1983), pp. 144-145.

7. R. Ulrich, in Proceedings of the NATO Advanced Study Institute on Optical Fiber Sensors (Nijhoff, Amsterdam, 1986), pp. 73-130.

8. A. S. Georges, F. Farahi, T. P. Newson, J. D. C. Jones, and D. A. Jackson, Int. J. Optoelectron. 13, 311 (1988).

9. G. Beheim, Appl. Opt. 24, 2335 (1985).

10. M. Lequime, C. Lecot, H. R. Giovannini, and S. J. Huard, Proc. Soc. Photo-Opt. Instrum. Eng. 1267, 288 (1990).

11. Th. Bosselman and R. Ulrich, in Proceedings of the 2nd International Conference on Optical Fiber Sensors (VDE-Verlag, Berlin, 1984), pp. 361-364.

12. C. Mariller and M. Lequime, Proc. Soc. Photo-Opt. Instrum. Eng. 298, 121 (1987). 